Music Listening as a Potential Aid in Reducing Emotional Eating.

\author{
Annemieke J. M. van den Tol ${ }^{1}$ \\ Helen Coulthard ${ }^{1}$ \\ Waldie E. Hanser ${ }^{2}$
}

1- De Montfort University, School of Applied Social Sciences, Leicester, United Kingdom

2 -Tilburg University, Department of Medical and Clinical Psychology, Tilburg, the Netherlands

Author Note: This research was conducted without any additional funding or grants.

Correspondence concerning this article should be addressed to Dr Annemieke J. M. van den Tol, Division of Psychology, De Montfort University, Hawthorn Building 0.35, Faculty of Health and Life Sciences, The Gateway, Leicester, LE1 9BH. Email: Annemieke.vandentol@ dmu.ac.uk. 


\begin{abstract}
Emotional Eating (EE) is understood as a maladaptive self-regulation strategy to satisfy emotional needs instead of hunger. Consequently, EE has been associated with negative health consequences. Enjoyment of food and music share similar neural activations in the brain and are both used by people for regulating affect. This suggests that music listening could potentially be a healthier alternative to EE. The present study was designed to investigate associations between EE, disordered mood, and music-related mood regulation.
\end{abstract}

Five-hundred-and-seventy-one participants completed measures of EE, music listening strategies and disordered mood. Associations between seven different music listening strategies and EE were examined, and also whether these regulation strategies were associated with depression, anxiety, and stress. Finally, we explored associations between music listening and EE in people with low and high (non-clinical) levels of disordered mood (depression, anxiety, and stress).

The findings of this research indicated that music listening for discharge (releasing anger or sadness through music that expresses these same emotions) and EE were positively associated with one another. In addition, EE and the music listening strategies of entertainment, diversion or mental work were associated in people with low levels of disordered mood. When disordered mood was high, EE was higher, but was not associated with music listening strategies. These associations point towards the possibility of some music listening strategies being useful as healthier alternatives for EE.

Keywords: music use, emotional eating, coping, emotion regulation, depression, anxiety, stress. 


\section{Music Listening as a Potential Aid in Reducing Emotional Eating: An exploratory study}

\section{Emotional Eating and listening to music}

Emotional eating (EE) can be defined as eating beyond the satiation point, or in the absence of hunger, in response to negative affect (e.g., Nguyen-Rodriguez, Unger \& Spruijt-Metz, 2009; Van Strien, Frijters, Bergers, \& Defares, 1986; Van Strien, 2002). Although EE may be a symptom of some eating disorder diagnoses in DSM-5 and ICD-10 such as Bulimia Nervosa and Binge Eating Disorder (American Psychiatric Association, 2013; World Health Organization, 2016), it is a specific behaviour rather than a clinical diagnosis and can exist along a continuum of severity (van Strien et al., 1986). More specifically EE occurs when an individual consumes more food than is physiologically necessary in a conscious or unconscious attempt to improve affect in the short term. EE has a high comorbidity with psychological problems such as depression and anxiety, and is more common in some individuals as a consequence of stress (Ouwens, Van Strien, \& Van Leeuwe, 2009; Nguyen-Rodriguez et al.,2009). EE is also associated with negative health outcomes as the overconsumption of snack foods is associated with increased caloric intake, obesity, weight gain, interference with weight loss (Hays \& Roberts, 2008; Koenders \& Van Strien, 2011; Konttinen, Männistö, Sarlio-Lähteenkorva, Silventoinen, \& Haukkala, 2010) and it is linked to chronic illnesses such as heart disease, cancer, and type-2 diabetes (Dixon, 2010).

A deeper understanding of the different psychological processes involved in emotional eating is of clinical importance, as it allows for the development of new behavioural therapies that teach people to regulate their emotions, and consequently, manage their caloric intake more effectively (Maratos, Gold, Wang, \& Crawford, 2008; Thaut, 2002). One potential mechanism that shares psychological features (reward and potential to regulate negative emotions) with eating, but is not associated with the same negative health outcomes, is music. It is possible that music listening can fulfil some of people's motivations for EE.

\section{Emotion regulation}

Emotion regulation refers to the processes involved in monitoring, evaluating or modifying emotional experience in order to accomplish personal goals (Thompson, 1994). Adaptive emotion regulation strategies are essential for mental (and sometimes physical) well-being (Gross, 2002). EE and the use of 
music for mood-regulation are both commonly used (in conscious or unconscious attempts) to regulate negative affect (Morris \& Reilly, 1987; Van Goethem \& Sloboda, 2011). A deeper understanding of this link could be useful in the management of EE. More specifically, information about the associations that EE has with various different music listening strategies may provide valuable first insights into how the initial motivation for engaging in EE can, potentially, be reduced by utilizing different music listening strategies.

\section{Emotional Eating}

Most theories share the assumption that when emotional eaters experience negative affect they cannot effectively regulate, they adopt an easily accessible strategy, like eating, to distract from (avoidant) or improve (emotion-oriented) negative emotions (Evers, Stok \& Ridder, 2010; Gibson, 2012; NguyenRodriguez et al., 2009). EE is associated with consumption of highly palatable snack foods (comfort foods), which are high in fat, sugar and salt, whilst being low in nutritional value (Van Strien et al., 2013). Empirical evidence indicates that the consumption of highly palatable foods protects against stress by the suppression of the hypothalamic-pituitary-adrenal axis response, although activation of the hypothalamicpituitary-adrenal axis may itself drive appetite for these foods in the long term (Gibson, 2012). Moreover, EE is seen as a maladaptive strategy to regulate mood, as it does not address the reason behind the negative emotions leading to repeated episodes (Spoor, Bekker, Van Strien, \& Van Heck, 2007) and often unwanted weight gain (Dixon, 2010).

EE can lead to feelings of guilt (Dubé, LeBel, \& Lu, 2005; Macht \& Dettmer, 2006) and emotional eaters show general patterns of emotion dysregulation, the need to escape from negative affect, and rumination (Gibson, 2012). Studies investigating antecedents of EE indicate that incidents that elicit low to moderately intense negative emotions, like daily hassles, are most likely to trigger EE (Macht, 2008). Hassles related to interpersonal problems which result in negative affect have been found to trigger of disturbed eating behaviour (Tanofsky-Kraff et al., 2007;Wilfley, Schwartz, Spurrell, \& Fairburn, 1997). These mechanisms explain why EE is sometimes referred to as comfort-eating or stress-eating (Gibson, 2012). 
Unlike food, music does not imply an obvious physiological satisfaction, but neuroimaging studies show activation of emotion, reward, motivation, and arousal networks during music listening (Blood \& Zatorre, 2001; Mas-Herrero, Marco-Pallares, Lorenzo-Seva, Zatorre, \& Rodriguez-Fornells, 2013

Salimpoor, Benovoy, Longo, Gooperstock, \& Zatorre, 2011). Music has been implicated as a versatile and effective tool to regulate emotions in a variety of studies (i.e., Saarikallio \& Erkkilä, 2007; Sloboda, Lamont, \& Greasley, 2009; Van den Tol \& Giner-Sorolla, 2016). It is believed (Schäfer, Sedlmeier, Städtler, \& Huron, 2013), that motivation for listening to music can partly be explained by its capacity to change, maintain or intensify affective states, and that this may even be the main reason for listening to music (e.g., Van Goethem \& Sloboda, 2011). Moreover, similar to EE, music is used habitually for mood regulating when in a negative mood (Randall \& Rickard, 2016). People also use music (and lyrics) for cognitive effects, including cognitive reappraisal, search for meaning, and for diversion from boredom, intrusive thoughts and emotions (see for example Saarikallio \& Erkkilä, 2007).

One important and well established framework for studying the use of music as a mood regulator is the Music in Mood Regulation Scale (MMR; Saarikallio, 2008). The following regulating strategies have been identified: entertainment (creating a nice atmosphere and happy feeling to maintain or enhance current positive mood and feeling), revival (personal renewal, relaxing, and getting new energy from music when stressed or tired), strong sensation (inducing and strengthening intense emotional experiences), diversion (using music to be distracted from negative thoughts and forgetting the current mood), discharge (releasing anger or sadness - similar to 'blowing off steam' - through music that expresses these same emotions), mental work (using music as a framework for mental contemplation and clarification of emotional preoccupations) and solace (searching acceptance and understanding when feeling sad or troubled). For the current work, we are interested in the association of EE with all of these seven different music listening strategies, as a higher association with EE may point towards a higher potential of interchangeable use.

Depression, Anxiety, and Stress

Links have been found between maladaptive affect-regulation strategies (such as extensive use of avoidant coping) and various psychological disorders including depression, anxiety, and stress (Berking et 
al., 2012; Cheng, Lau, \& Chan, 2014), in which unhealthy patterns of regulating emotions are often present before the onset of such disorders. Moreover, the associations between EE and all different music listening strategies might also be dependent on a person's existing level of disordered mood (low vs. high). As indicated earlier, EE has comorbidity with disordered mood. Results from an experimental study where snack foods were provided in a sham taste test showed that trait anxiety led to higher food intake following a mood induction in obese participants (Schneider, Appelhans, Whited, Oleski, \& Pagoto, 2010). Similarly, EE has been found to be associated with depression (Konttinen et al., 2010; Ouwens et al., 2009) and stress (Nguyen-Rodriguez et al., 2009; Nolan \& Geliebter, 2012) when measured through self-report.

Certain music listening strategies have also been linked to disordered mood. The use of music for discharge has, for example, been related to depression, anxiety and neuroticism in several self-report studies (Carlson et al., 2015; Thomson, Reece, \& Di Benedetto, 2014; Chin \& Rickard, 2013). Thomson and others (2014) also found that using music for distraction (a concept closely related to the MMR diversion subscale) correlated with anxiety and stress. Chin and Rickard (2013) reported an additional negative association between entertainment, strong sensation and disordered mood (depression, anxiety, and stress), and found that relationships with disordered mood can partly be explained by those with better mental health using less suppression.

Unlike EE, for which associations with mental health are mainly seen as an indicator of maladaptive coping, research about music listening suggest that one reason why some associations exist with disordered mood might actually be because music listening can help one cope with disorder mood (Getz, Marks, \& Roy, 2014, Van den Tol \& Edwards, 2013). People who are exposed to a stressor in an experiment are, for example, more likely to want to listen to music (Getz et al., 2014). Moreover, participants in a retrospective survey study reported that they were motivated to listen to sad music when feeling sad in order to accept negative situations and work through emotions (Van den Tol, Edwards \& Heflick, 2016). Several experimental studies in which participants have been subjected to a stress induction and then have listened to self-selected music have shown decreased state anxiety, improved negative mood, and reduced stress compared to non music control group (Getz et al., 2014; Labbé, Schmidt, Babin, \& Pharr, 2007; Radstaak, Geurts, Brosschot, \& Kompier, 2014). 
This research aims to identify what music listening strategy is most strongly associated with tendencies for EE, as this would provide future indicators (in terms of receive extra attention in future research) of which might be the more adaptive music listening strategies (in terms of long term mental health) for replacing EE.

\begin{abstract}
Aims
The current study has two main aims: To examine associations between music listening strategies, disordered mood (depression, anxiety, stress) and EE. To identify potential interactions between disordered mood (in terms of high and low levels of depression, anxiety, and stress) and use of music selection strategies in relation to EE.
\end{abstract}

The following research questions were focal in trying to pursue these aims: (1) Which (if any) music listening strategies, are associated with EE?, and 2) Do associations between music listening strategies and EE vary for high or low disordered mood?

\title{
Method
}

\section{Participants}

Eight hundred participants were recruited, using opportunity sampling from De Montfort University campus, and online through social media websites via a short advertisement and link to the questionnaire. Participants were required to be fluent in English to ensure item comprehension, and to be at least 18 years of age. Two hundred and thirty participants were excluded due to not meeting inclusion criterion (English as a first language), withdrawal, failure to properly indicate consent and incomplete data.

The final sample consisted of 570 participants (See Table 1), comprised of 188 (33.40\%) males, 375 females $(65.8 \%)$ and $7(1.2 \%)$ participants who preferred not to disclose their sex, with a mean age $(N=569$, $18-65)$ of $23.41(S D=4.39)$. The average indicated height $(N=565)$ was $169.62 \mathrm{~cm}(S D=10.76$, ranging from 134 to $200 \mathrm{~cm})$ and the average indicated weight $(N=564)$ was $69.36 \mathrm{~kg}(S D=18.45$, ranging from 40 to 181). Body Mass Index (BMI) average (for those participants that provided both weight and height $(N=557)$ was calculated to be $23.92(S D=5.46$, ranging from 15.05 to 58.00). The participants in our 
sample had a mean EE score of $2.5(S D=0.8)$, which was similar to that of several recent studies (2.7 (SD $=0.7)$ in Anschutz, van Strien, Van den Ven, \& Engels, 2009; 2.4(SD = 0.9) in Van Strien et al., 2013). We did not collect data on participants' nationality or socioeconomic background, however we know that this was an educated University sample. Moreover, De Montfort University has a diverse population of students; 43\% are white-European and 57\% are from BAME (black and minority ethnic) backgrounds, including $24 \%$ black African/ Caribbean and 17\% South Asian.

\section{PLEASE INSER TABLE 1 HERE}

\section{Design and Materials}

The study had a cross-sectional, correlational design. The dependent variable was emotional eating (EE), the independent variables were negative mood (depression, anxiety and stress) and the moderator variables were the music listening for mood regulation variables (entertainment, revival, discharge, diversion, solace, mental work, and strong sensation).

\section{The Dutch Eating Behaviour Questionnaire}

EE was measured with the English version (Wardle, 1987) of the 13-item EE subscale of the Dutch Eating Behaviour Questionnaire (DEBQ; Van Strien et al., 1986). The sub-scale includes 13 items (e.g., Do you have a desire to eat when you are feeling lonely?) on a 5-point Likert scale $(1=$ Never, $5=$ Very Often $)$. Cronbach's alpha for the sub-scale was good at .91 in the current sample.

Music in Mood Regulation Scale

Music related mood-regulating strategies were measured with the 40 item Music in Mood Regulation scale (MMR; Saarikallio, 2008). Items are rated on a 5-point Likert scale ( $1=$ Strongly Disagree, 5= Strongly Agree). All seven sub-scales were used: (1) Entertainment (4 items, e.g., I usually put background music on to make the atmosphere more pleasant), (2) Revival (7 items, e.g., Listening to music helps me to relax), (3) Diversion (5 items, e.g., For me, music is a way for me to forget my worries), (4) Discharge (6 items, e.g., When everything feels miserable, I start to listen to music that expresses these feelings), (5) Strong sensation (7 items, e.g., Music offers me unforgettable moments), (6) Mental work (5 
items, e.g., Listening to music takes me back and gets me thinking about different things that have happened to me), and (7) Solace (6 items, e.g., When something is troubling me, I find solace in music). The scale has a Cronbach's Alpha of .95 in the current sample, and reliability ranges from .78 to .86 for each sub-scale.

Depression, Anxiety, and Stress.

Depression, anxiety, and stress were measured with the 42-item Depression, Anxiety, and Stress Scales (DASS; Lovibond \& Lovibond, 1995). Each sub-scale of the DASS consists of 14 items and measure depression (e.g., I felt down-hearted and blue), anxiety (e.g., I felt scared without any good reason), and stress (e.g., I was in a state of nervous tension). We removed the eating-behaviour item (I had difficulty in swallowing) in the depression scale, as it would distort the correlation with the EE sub-scale. For each item participants rated the extent to which they experienced negative emotional symptoms over the past week on a 5-point severity/ frequency scale $(0=$ did not apply at all, 4=applied very much, or most of the time $)$, while this scale is traditionally rated on a 0 to 3 Likert scale. The sub-scales' Cronbach's alpha in the current sample range from .95 to .97 and all sub-scales have well-established psychometric properties in community samples (Bayram \& Bilgel, 2008).

\section{Procedure}

Participants were recruited online and through opportunity sampling. The online survey software 'www.Qualtrics.com' was utilised to collect data online from participants. Ethical guidelines set out by the British Psychological Society (2013a, 2014) were adhered to throughout the research process, and ethical approval was granted by the Faculty Research Ethics Committee (FREC) at De Montfort University.

\section{Preliminary analysis}

Data analysis was completed using IBM SPSS 22 with a Process add-on (Hayes, 2013) for moderation analyses. Initial normality tests indicated that several variables were not normally distributed, therefore 1000 bootstrapped corrected confidence intervals (95\%) were used for all linear regression analyses. Previous research suggested the possibility of age, gender and BMI differences in music listening, and disordered mood (Carlson et al., 2015; Crawford \& Henry, 2003; Schoevers, Beekman, Deeg, Jonker, \& Van Tilburg, 2003; Thomson et al., 2014; Larsen, Van Strien, Eisinga, \& Engels, 2007). A MANOVA did reveal a significant difference for gender (Wilks' $\Lambda=.90, F(11,551)=5.459, p<.001, \quad{ }_{p}^{2}$ with $=.10$, 
medium effect size). With post-hoc tests (Table 1) revealing a gender difference for EE, and stress (at small effect size, see Table 1). None of the variables yielded a significant association with age. Stress and EE were positively associated with BMI. Analyses therefore controlled for gender and BMI where relevant (for all analyses in which either EE or stress was the dependent variable).

\section{Results}

(Aim 1) associations between music listening strategies, disordered mood (depression, anxiety, stress) and $E E$.

A series of linear regression analyses was conducted to examine associations between EE, music listening strategies and disordered mood (depression, anxiety, stress). Either EE (Table 2a) or one of the disordered mood variables (Table $2 b$ ) was the DV in all analyses. Music listening strategies or disordered mood were the IV in analyses where EE was the DV, and music listening strategies were the IV in analyses where disordered mood was the DV.

The regression analyses controlled for gender and BMI when either EE or stress were the DV, but not when depression or anxiety was the DV. One thousand bootstrapped corrected confidence intervals $(95 \%)$ were used for all linear regression analyses to adjust for any issues with normality in the data.

As can be seen in Table 2a, EE was only related to one music regulation strategy, discharge, but was associated with all the disordered mood variables (depression, anxiety, and stress). As can be seen in Table 2b, discharge and solace were associated with depression, anxiety, and stress. Moreover, strong sensation was associated with depression and stress, and mental work with depression and anxiety. We did not find any other significant association for any of the music listening strategies with disordered mood.

\section{PLEASE INSERT TABLE 2A AND 2B HERE}

(Aim 2) Identifying potential interactions between disordered mood (in terms of low and high levels of depression, anxiety, and stress) and use of music selection strategies in relation to EE.

To examine whether music listening strategies moderated the relationship between mood and EE a series of 21 moderated regressions was conducted using Process Model 1 (Hayes, 2013). Depression, 
anxiety, and stress were entered individually, one in each analysis, as an independent variable, along with one of the seven music selection strategies as the moderator. EE was entered as the dependent variable in all analyses. When significant, the interaction was then further tested, with each independent variable set to + or $-1 \mathrm{SD}$ from the mean.

\section{PLEASE INSERT TABLE 3 AND FIGURE 1 HERE}

The analyses provided a total of six significant interaction effects (see Table 3 for an overview of all statistics); entertainment and depression, entertainment and stress, diversion and depression, diversion and stress, mental-work and depression, and mental-work and stress. As can be seen in Table 3 (also see Figure 1 for a visual illustration of this pattern), all six interaction effects yielded a similar pattern: At high (+1 SD) levels of disordered mood there was no association between music listening strategy and EE. At low (-1 SD) levels of disordered $\operatorname{mood}^{1}$ there was always a positive association between music listening strategy and EE (this effect was significant for all four analysis in which either entertainment or diversion was present, was marginally significant for the moderation with mental work and depression, and was approaching significance for the moderation analysis with mental work and stress, see Table 3 for statistics). In particular low levels of music listening strategy were linked to low EE, and high levels of music listening strategy were linked to high EE.

To explain these findings once more in terms of mean EE scores. All six interaction effects yielded a similar pattern: EE was higher when there was a high level of disordered mood, regardless of music listening. EE was lowest when people scored both low on using music as a strategy and score low on disordered mood (also see Figure 1 for one example of a visual illustration of this pattern, which was similar across all 6 interactions).

\section{Discussion}

The first aim of this research was to examine associations between music listening strategies, disordered mood (depression, anxiety, stress) and EE. Current data showed an association between discharge 
and EE. Moreover, depression, anxiety and stress were all associated with EE. It is also found that discharge, and solace were all associated with depression, anxiety, and stress. The use of music for strong sensations was additionally associated with depression and stress (but not with anxiety). The use of music for mental work is additionally associated with anxiety and depression (but not with stress).

The second aim of this research was to identify potential interactions between (high and low levels of) disordered mood (in terms of depression, anxiety, and stress) and use of music selection strategies in relation to EE. In the current study it was found that levels of EE were dependent on a combination of disordered mood and music use for; entertainment and depression, entertainment and stress, diversion and depression, diversion and stress, mental work and depression, and mental work and stress. For these six combinations of variables there was no difference in levels of EE depending on music use for high values of disordered mood, but EE was higher when music use was higher for low values of disordered mood.

In the next two sections of the discussion all four music listening strategies that are related to EE will be discussed (overall or at either low or high levels of disordered mood). Firstly the association between emotional eating and discharge will be discussed, followed by the associations that EE had to music listening at low levels of disordered mood (depression and stress); music listening for diversion, entertainment, and mental work.

\section{Discharge}

One of the most central findings in this research was that EE was associated with the music listening strategy discharge. This suggest that discharge mimics EE. One important question that this association raises is whether music listening for discharge can also be used to reduce, or as an alternative for, EE? Considering the nature of discharge, there are two possible short-term outcomes of using discharge in order to reduce motivation to engage in EE: One possible outcome may be that people may continue to wallow in their negative mood and therefore may still feel the urge to eat. This is assuming that participants do not experience a cathartic release of negative mood after listening to music for discharge, which to our knowledge has not been tested yet in experimental research. However, in two experimental studies (Labbé et al., 2007; Getz et al., 2014) in which participants listened to (experimenter selected) metal music (many of the items on discharge are about the use of angry music) it has been found that people who experience stress 
(exam stress, or an experimental stress induction) did not feel better after doing so. Saying this, being in touch with sad or negative emotions (an element of using discharge music) might actually help people to accept negativity and enable people to move on (Van den Tol et al., 2016) and feel better, especially when people would otherwise supress such negative emotions (Chin \& Rickard, 2013). The other possible outcome is that people do feel relieved after listening to self-chosen discharge music and that the motivation to engage in EE disappears as a result. Our self-report data however does not provide any evidence about EE having the potential to replace EE and further experimental and behavioural research is needed to explore such a causal effect.

\section{Is discharge a good alternative to EE?}

As suggested earlier, music listening could be a healthy alternative to EE in terms of physical health, however is music listening for discharge also a healthier alternative in terms of mental health? In this research it was found that both $\mathrm{EE}$ and discharge were associated with depression, anxiety and stress. Although there can be no certainty about whether these associations are the result of maladaptive coping (using the wrong coping strategy in the wrong situation), EE and discharge have both been associated with ruminative tendencies and dysregulation in previous research (Carlson et al., 2015; Gibson, 2012; NolenHoeksema, 1991; Silk, Steinberg, \& Morris, 2003) and both have a component of being overwhelmed by t negative emotional experience, and of 'letting oneself go' in terms of emotions. In addition, depression, anxiety, and stress are all conditions in which people are overwhelmed by negative mood, and may experience increased ruminating (Lovibond \& Lovibond, 1995). Therefore, it seems likely that some of these overlapping mechanisms are important in explaining the relationship between EE and discharge and their correlations with depression, anxiety and stress. It is possible therefore that discharge may generally not be such a good alternative for replacing EE in terms of mental health. Future research should look further into which one of these concepts (being overwhelmed, letting go in terms of ones emotions, or ruminating) is most important for each association, in order to find out more about the long-term consequences of listening to music for discharge.

Future research should examine further the long-term effects of listening to music on mental health before concluding whether or not music should be used as a tool to decrease EE. Results of a recent study 
conducted on people's private phone with a music app (Randall, Rickard, \& Vella-Brodrick, 2014) revealed that using music to regulate a recently experienced emotion yielded the greatest hedonic success, but was associated with poorer emotional health and well-being. Research has additionally indicated that the use of music for suppression of a negative mood is associated with lower levels of mental health (Chin \& Rickard, 2013), meaning that suppression of a negative mood might not be a good coping strategy. Related to this, Gortner, Rude and Pennebaker (2014) found a decrease in rumination and depression in a study in which participants were asked to regularly write about negative life events (thus regularly expressed their negative feelings and thoughts). These studies suggest that expressive strategies such as discharge may be advantageous for long term mental health when used responsibly.. In sum, in order to understand how adaptive or maladaptive the use of music for discharge is to decrease EE, future research would need to consider both short term mood effects and long term mental health effects.

\section{Music listenings' associations with EE at low disordered mood}

Findings indicated a positive association with EE for diversion, entertainment, and mental work, when particularly looking at those participants who were scoring low on depression and stress. This is especially interesting when we consider the fact that these moderated associations are mainly driven by people who score low on stress or depression. None of these music listening strategies was associated with stress, and two of these music listening strategies (diversion and entertainment) were not associated to depression or anxiety. Moreover, the use of music for diversion and entertainment have clearly been related to enhancing mood or maintaining positive mood (Van den Tol \& Giner-Sorolla, 2016), whereas the use of music for mental work regards contemplation and clarification of emotional preoccupations (Saarikallio, 2008) which is a process that can free a person of negative affect (Chin \& Rickard, 2013; Van den Tol, 2016).

The assumption that all of these music listening strategies are considered adaptive for relieving a bad mood might potentially point towards music already being used by some people in an attempt to counter impulses to engage in EE. Moreover, it might point towards this being a healthy coping strategy, as those people with better mental health might use more adaptive coping styles in general (Berking et al., 2012; 
Cheng et al, 2014). Thus although these people do engage in EE they are trying to reduce this impulse by using music listening strategies that reduce the negative moods associated with EE and by trying to maintain or enhance any existing positive mood (hence avoiding the urge to engage in $\mathrm{EE}$ ).

A literature review on flexible coping (Cheng et al., 2014) does show that people with better mental health do use a larger variety of activities (such as music, eating, calling a friend etc.) as coping strategies. Those with better mental health might therefore be more likely to use both (or even simultaneously engage in) $\mathrm{EE}$ and music listening strategies as a mean to cope with problems, creating the higher association with EE among lower mental health (and potentially even partly creating the lower association of EE for lower disordered mood). Thus people with higher disordered mood (worse mental health) might be more likely to only use a coping strategy (EE) which results in negative health outcomes (e.g. excessive calorie intake, weight gain) and not try to counter their negative mood with the use of music.

An alternative explanation might be that people low and high in disordered mood engage in EE and/or music listening for different reasons. To give an example, both $\mathrm{EE}$ and diversion are being described as being used for distraction from (or avoidance of) negative emotions (Evers et al., 2010; NguyenRodriguez et al., 2009 Saarikallio, 2008). While people low in depression might use EE for this reason, people high in disordered mood might have different motivations for EE. Thus although both engage in EE and music listening, both do so for different reasons meaning that we find different associations for both groups.

Is diversion, entertainment, or mental work a more promising music listening strategy for reducing EE?

One of the areas of interest in the current research was to examine which music listening strategy might potentially be used as a healthier alternative to EE. There has already been a discussion about discharge in this role. The next sections will discuss diversion, entertainment or mental work in this role.

\section{Diversion}

Diversion refers to the strategy of using music to distract from negative thoughts and forget the current mood (Saarikallio, 2008). This description is in line with the description of some of the episodes of EE (Evers et al., 2010; Nguyen-Rodriguez et al., 2009) as an attempt to distract from (avoidance) or improve 
(emotion-oriented) a negative mood. This explains why diversion might potentially be used by some people who engage in EE. Thus using diversion for reducing EE might potentially be beneficial in terms of reducing the negative mood which creates the impulse to overeat among EEs. As we cannot (yet) be equally sure if this will also be the case for discharge, it is possible that using diversion rather than discharge might yield better results in terms of reducing EE in the short term. That being said, like EE this strategy does not help one to actually solve the problems that are causing the negative moods in the first place.

\section{Entertainment}

Using music for entertainment has been described as creating a nice atmosphere and pleasant emotions to maintain or enhance positive mood (Saarikallio, 2008). This association with EE maybe explained by a more positive overlap with EE, in terms of EE being an attempt to distract from (avoidance) or improve (emotion-oriented) a negative mood (Evers et al., 2010; Nguyen-Rodriguez et al., 2009). This overlap can be considered rather promising in terms of reducing the motivation for EE, as EE is generally associated with a prior negative mood, rather than a positive mood.

Moreover, entertainment might also be used as a preventative measure against negative mood, which might explain why it is not associated with depression, anxiety, or stress in the current sample. Based on these considerations entertainment seems a very promising alternative to EE. However, people with higher levels of disordered mood might feel less motivated to engage in entertainment, as they may avoid positive experiences (Lovibond, \& Lovibond, 1995). Future research should explore how effective entertainment is in terms of reducing motivation to engage in $\mathrm{EE}$.

\section{Mental work}

Mental work refers to music being used as a framework for mental contemplation and clarification of emotional preoccupations (Saarikallio, 2008) which suggests a more analytical approach and problem focused coping style. As mental work can reduce stress and negative mood (Chin \& Rickard, 2013; Van den Tol \& Edwards, 2015) it seems like a good alternative to reduce EE impulse in the short term. As it additionally does not relate to avoiding (such as diversion) or suppressing (such as might be the case for discharge) mood (Chin \& Rickard, 2013) it might additionally be a promising long term strategy. However we cannot be sure about what the long term effects of using this strategy to replace EE would be in terms of 
mental health. Mental work may lead to the conclusion that a situation cannot be resolved and goals may have to be adjusted. Mental work might hence reduce direct stress levels (i.e., 'If I can't solve it why would I be stressed') but might increase overall depression and anxiety levels as reflected by the current association which mental work has with depression and anxiety but not with stress. Longitudinal future research should look into this.

\section{A final note about these moderated effects}

As a final note about these moderations, we want to warn readers not to confuse 'the relations between EE and music listening strategies 'among' low or high depression and stress' with 'the relations of EE and music listening strategies 'with' depression and stress'. Thus, even though EE and mental work are both associated with disordered mood, the associations between mental work and EE were nevertheless higher for people low on stress and depression (as these two things are different). As indicated earlier, although maladaptive use of coping is linked to mental health problems (Berking et al., 2012; Cheng et al., 2014) associations of certain coping strategies with disordered mood do not necessarily indicate that these coping strategies 'caused' the mental health problems. It might just as well be possible that people with more mental health problems are more likely to use a certain strategy because it actually helps them to cope with those mental health problems. The interactions found in this research for EE and mental work among lower disordered mood might potentially point towards this being the case here.

\section{Limitations and future research}

There are a number of strengths associated with this study. Our collected data were drawn from a large sample of participants who were blind to the real purpose of the study. Many previous studies that have been carried out on the topic of music were advertised either on the radio or advertised by music students as being a study about music, hence drawing upon a population that are more likely to be musicians or have music as an important aspect of their life (Carlson et al., 2015; Hanser, Ter Bogt, Van den Tol, Mark, \& Vingerhoets, 2016; Van Goethem \& Sloboda, 2011).

One limitation of this research is the use of self-report data. Although the self-report measures used are considered to be valid for depression, anxiety, and stress (Antony, Bieling, Cox, Enns, \& Swinson, 
1998), EE is a more difficult behaviour to measure, as scores on EE measures and actual eating behaviour are not always associated (Larsen, Van Strien, Eisinga, Herman, \& Engels, 2007). Future research should examine the mechanisms behind the associations between regulation using music and food, for example by measuring the relationship between music and food intake after experimentally inducing real life emotions, or naturalistically documenting people's music and eating behaviour in real life (with the help of technological interventions). In particular, such research should test if the maladaptive strategy of EE can be reduced by the use of music listening strategies and which strategies are the most effective disruptors of EE behaviours. In addition, future research might also want to look at links between music listening and its association with different facets of EE (within and beyond the DEBQ) and at long term mental health effects of using certain music listening strategies in order to replace EE.

The current data that was gathered on depression, anxiety, and stress, measured recent episodes (i.e., last two weeks), but the questions on affect regulation strategies measured people's general tendencies (i.e., time not specified). Research (Tearne, Jianghong, \& McLean, 2016) has indicated that the DASS scale has stability over substantial periods of time (3 to 8 years). Nevertheless it is possible that associations would have been stronger (or weaker) if we would have either asked all questions with regards to the last two weeks or as a general tendency.

Another limitation of the current research is that there is not a simple relationship between negative mood and over eating; some people eat fewer foods following negative life events, whilst other people will over-eat in response to positive affect (Geliebter \& Aversa, 2003). We did not measure these tendencies in the current study and therefore cannot be sure if these have impacted on our results in any way. The tendency to under-eat in response to stress is linked with lower BMI (Tuschl, 1990). Our research did however control for BMI across all analyses. Future studies could look exclusively at individuals who over eat in response to negative emotions, and exclude other emotional eating styles.

There have been some recent criticisms of questionnaire and laboratory measures of EE (see Bongers \& Jansen, 2016), which must be acknowledged as part of the evaluation of this study. It has been proposed that EE may be part of cluster of behaviours related to uncontrolled eating (Vainik, Neseliler, Konstabel, Fellows, \& Dagher, 2015), characterised by low perceived self-control and a strong desire to eat. In addition, 
it has also been stated that individuals who have high scores on EE scales, may actually be 'concerned eaters' who worry about their eating behaviour and their self-control (Jansen et al., 2011). It could be that the association between listening to music for discharge and EE may be caused by low perceived selfcontrol, and this would be an interesting area for future research.

\section{Implications}

This paper examined and founnd associations between EE and music listening strategies in relation to depression, anxiety, and stress, bringing together two distinct areas of emotional regulation research. The findings indicate that music listening and EE are especially associated for people with low levels of disordered mood (when it regards listening to music for entertainment, distraction or mental work), or when music is used for discharge (which regards releasing anger or sadness through music that expresses these same emotions). These associations point towards the possibility of some music listening strategies being useful as a physiologically, but also mentally, healthier alternative for EE. As this was a correlational design, it can only be concluded that different constructs are associated with each other, not what the mechanisms of those relationships are. This research has the potential to lead to further experimental and longitudinal studies, to examine whether adaptive music listening strategies can disrupt the association between negative mood and EE and if this will be beneficial in terms of people's mental health in the long term.

\section{Acknowledgements}

We would like to acknowledge Melanie Ward, Haley Fong, \& Michael Buccheri for helping us gather the data for this research and entering all data in SPSS. 


\section{References}

American Psychiatric Association. (2013). Diagnostic and statistical manual of mental disorders: DSM-5 (5th Ed.). Washington, DC: American Psychiatric Association.

Antony, M. M., Bieling, P. J., Cox, B. J., Enns, M. W., \& Swinson, R. P. (1998). Psychometric properties of the 42item and 21-item versions of the depression anxiety stress scales in clinical groups and a community sample. Psychological Assessment, 10(2), 176-181.

Anschutz, D. J., Van Strien, T., Van de Ven, M. O. M., \& Engels, R. C. (2009). Eating styles and energy intake in young women. Appetite, 53(1), 119-122.

Baste, V. S., \& Gadkari, J. V. (2014). Study of stress, self-esteem and depression in medical students and effect of music on perceived stress. Indian Journal of Physiology and Pharmacology, 58(3), 296-299.

Bayram, N., \& Bilgel, N. (2008). The prevalence and socio-demographic correlations of depression, anxiety and stress among a group of university students. Social Psychiatry and Psychiatric Epidemiology, 43(8), 667672.

Berking, M., Poppe, C., Luhmann, M., Wupperman, P., Jaggi, V., \& Seifritz, E. (2012). Is the association between various emotion-regulation skills and mental health mediated by the ability to modify emotions? Results from two cross-sectional studies. Journal of Behavioral Therapy and Experimental Psychiatry, 43(3), 931937.

Blood, A. J., \& Zatorre, R. J. (2001). Intensely pleasurable responses to music correlate with activity in brain regions implicated in reward and emotion. Proceedings of the National Academy of Sciences, 98(20), $11818-11823$.

Bongers, P., \& Jansen, A. (2016). Emotional eating is not what you think it is and emotional eating scales do not measure what you think they measure. Frontiers in Psychology, 43(3), 931-937.

Carlson, E., Saarikallio, S., Toiviainen, P., Bogert, B., Kliuchko, M., \& Brattico, E. (2015). Maladaptive and adaptive emotion regulation through music: a behavioral and neuroimaging study of males and females. Frontiers in Human Neuroscience, 43(3), 931-937. 
Chin, T., \& Rickard, N. S., (2013). Emotion regulation strategy mediates both positive and negative relationships between music uses and wellbeing. Psychology of Music, 42(5), 692-713.

Cheng, C., Lau, H. B., \& Chan, M. S. (2014). Coping flexibility and psychological adjustment to stressful life changes: a meta-analytic review. Psychological Bulletin.140, 1528-1607.

Crawford, J. R., \& Henry, J. D. (2003). The Depression Anxiety Stress Scales (DASS): Normative data and latent structure in a large non clinical sample. British Journal of Clinical Psychology, 42, 111-131.

Dixon, J. B. (2010). The effect of obesity on health outcomes. Molecular and Cellular Endocrinology, 316(2), 25, 104-108.

Dubé, L., LeBel. J.L., \& Lu, J. (2005). Affect asymmetry and comfort food consumption. Physiological Behavior, $86,559-567$.

Evers, C., Stok, F. M., \& De Ridder, D. T. D. (2010). Feeding your feelings: Emotion regulation strategies and emotional eating. Personality and Social Psychology Bulletin, 36(6), 792-804.

Geliebter, A., \& Aversa, A. (2003). Emotional eating in overweight, normal weight, and underweight individuals. Eating Behaviors, 3, 341-347.

Getz, L. M., Marks, S., \& Roy, M. (2014). The influence of stress, optimism, and music training on music uses and preferences. Psychology of Music, 42(1), 71-85.

Gibson, E. L. (2012). The psychobiology of comfort eating: implications for neuropharmacological interventions. Behavioural Pharmacology, 23(5 and 6), 442-460.

Gortner, E. M., Rude, S. S., \& Pennebaker, J. W. (2006). Benefits of expressive writing in lowering rumination and depressive symptoms. Behavior Therapy. 37(3), 292-303.

Gross, J. J. (2002). Emotion regulation: Affective, cognitive, and social consequences. Psychophysiology, 39(3), $281-291$

Hanser, W. E., Ter Bogt, T. F. M., Van den Tol, A. J. M. Mark., R. E., \& Vingerhoets, A. J. J. M. (2016). Consolation through music: A survey study. Musicae Scientiae, 20 (1), 81-101.

Hayes, A. F. (2013). Introduction to mediation, moderation, and conditional process analysis: A regression-based approach. New York: Guilford Press. 
Hays, N. P., \& Roberts, S. B. (2008). Aspects of eating behaviors “disinhibition" and "restraint" are related to weight gain and BMI in women. Obesity, 16, 52-58.

Jansen, P. W., Roza, S. J., Jaddoe, V. W., Mackenbach, J. D., Raat, H., Hofman, A., Verhulst F. C., \& Tiemeier, H. (2012). Children's eating behavior, feeding practices of parents and weight problems in early childhood: results from the population-based Generation R Study. International Journal of Behavioural Nutrition and Physical Activity, 9, $130-141$.

Koenders, P. G., \& Van Strien, T. (2011). Emotional eating, rather than lifestyle behavior, drives weight gain in a prospective study in 1562 employees. Journal of Occupational and Environmental Medicine, 53(11), $12-87$. Konttinen, H., Männistö, S., Sarlio-Lähteenkorva, S., Silventoinen, K., \& Haukkala, A. (2010). Emotional eating, depressive symptoms and self-reported food consumption. A population-based study. Appetite, 54(3), 473479.

Labbé, E., Schmidt, N., Babin, J., \& Pharr, M. (2007). Coping with stress: the effectiveness of different types of music. Applied Psychophysiology and Biofeedback, 32(3-4), 163-168.

Larsen, J. K., Van Strien, T., Eisinga, R., \& Engels, R. C. M. E. (2007). Gender differences in the association between alexithymia and emotional eating in obese individuals. Journal of Psychosomatic Research, 60(3), 237-243.

Larsen, J. K., Van Strien, T., Eisinga, R., Herman, C. P., \& Engels, R. C. M. E. (2007). Dietary restraint: intention versus behavior to restrict food intake. Appetite, 49(1), 100-108.

Lovibond, S. H., \& Lovibond, P. F. (1995). Manual for the depression anxiety stress scales (2nd ed.). Sydney, AU: Psychology Foundation of Australia.

Macht, M. (2008). How emotions affect eating: a five-way model. Appetite, 50, 1-11.

Macht, M., \& Dettmer, D. (2006). Everyday mood and emotions after eating a chocolate bar or an apple. Appetite, $46,332-336$

Maratos, A. S., Gold, C., Wang, X., \& Crawford, M. J. (2008). Music therapy for depression. Cochrane Database Systematic Review.

Mas-Herrero, E., Marco-Pallares, J., Lorenzo-Seva, U., Zatorre, R. J., \& Rodriguez-Fornells, A. (2013). Individual differences in music reward experiences. Music Perception, 31(2), 118-138. 
Morris, W. N., \& Reilly, N. P. (1987). Toward the self-regulation of mood: Theory and research. Motivation and Emotion, 11(3), 215-249.

Nguyen-Rodriguez, S. T., Unger, J. B., \& Spruijt-Metz, D. (2009). Psychological determinants of emotional eating in adolescence. Eating Disorders, 17(3), 211-224.

Nolan, L. J., \& Geliebter, A. (2012). Night eating is associated with emotional and external eating in college students. Eating Behaviors, 13, 202-206.

Nolen-Hoeksema, S. (1991). Responses to depression and their effects on the duration of depressive episodes. Journal of Abnormal Psychology, 100(4), 569-582.

Ouwens, M. A., Van Strien, T., \& Van Leeuwe, J. F. J. (2009). Possible pathways between depression, emotional and external eating. A structural equation model. Appetite, 53(2), 245-248.

Radstaak, M., Geurts, S. A., Brosschot, J. F., \& Kompier, M. A. (2014). Music and psychophysiological recovery from stress. Psychosomatic Medicine, 76(7), 529-537.

Randall, W. M., \& Rickard, N. S., (2016). Reasons for personal music listening: A mobile experience sampling study of emotional outcomes. Psychology of Music, 45(4), 479 - 495.

Randall, W. M., Rickard, N. S., \& Vella-Broderick, D. A. (2014). Emotional outcomes of regulation strategies used during personal listening: A mobile experience sampling study. Musicae Scientiae, 18(3), 275-291.

Saarikallio, S. (2008). Music in mood regulation: Initial scale development. Musicae Scientiae, 12(2), 291-309.

Saarikallio, S., \& Erkkilä, J. (2007). The role of music in adolescents' mood regulation. Psychology of Music, 35(1), 88-109.

Salimpoor, V. N., Benovoy, M., Longo, G., Cooperstock, J.R., \& Zatorre, R.J. (2011). The rewarding aspects of music listening are related to degree of emotional arousal. PLoS ONE, 4, 74 - 87.

Schäfer, T., Sedlmeier, P., Städtler, C., \& Huron, D. (2013). The psychological functions of music listening. Frontiers in Psychology, 4, 511-225.

Schneider, K. L., Appelhans, B. M., Whited, M. C., Oleski. J., \& Pagoto, S. L. (2010). Trait anxiety, but not trait anger, predisposes obese individuals to emotional eating. Appetite. 55, 701-706. 
Schoevers, R. A., Beekman, A. T. F., Deeg, D. J. H., Jonker, C., \& Van Tilburg, W. (2003). Comorbidity and riskpatterns of depression, generalised anxiety disorder and mixed anxiety-depression in later life: results from the AMSTEL study. International Journal of Geriatric Psychiatry, 18(11), 1099-1166.

Silk, J. S., Steinberg, L., \& Morris, A. S. (2003). Adolescents' emotion regulation in daily life: Links to depressive symptoms and problem behavior. Child Development, 74, 1869-1880.

Sloboda, J. A., Lamont, A., \& Greasley, A. (2009). Choosing to hear music: Motivation, process and effect. In Hallam S., Cross I., Thaut M. (Eds.), The Oxford handbook of music psychology (pp. 431-440). Oxford, UK: Oxford University Press.

Spoor, S. T. P., Bekker, M, H. J., Van Strien, T., \& Van Heck, G. L. (2007). Relations between negative affect, coping, and emotional eating, Appetite, 48(3), 368-376.

Tanofsky-Kraff, M., Wilfley, D. E., Young, J. F., Mufson, L., Susan, Z., Yanovski, D. R., Glasofer, \& Salaita, C. G. (2007). Preventing excessive weight gain in adolescents: interpersonal psychotherapy for binge eating. Obesity, 15(6), 1345-1355.

Tearne, J. E., Jianghong, L., \& McLean, N. J. (2016). Older maternal age is associated with depression, anxiety, and stress symptoms in young adult female offspring. Journal of Abnormal Psychology, 125, 1, 1-10.

Thaut, M. H. (2002). Toward a cognition-affect model in neuropsychiatric music therapy. In R. F. Unkefer \& M. T. Thaut (Eds.), Music therapy in the treatment of adults with mental disorders: Theoretical bases and clinical interventions (pp. 86-103). Gilsum, NH: Barcelona Publishers.

Thompson, R. A. (1994). Emotion regulation: A theme in search of definition. Monographs of the Society for Research in Child Development, 59(2-3), 25-52.

Thomson, C. J., Reece, J. E., \& Di Benedetto, M. (2014). The relationship between music-related mood regulation and psychopathology in young people. Musicae Scientiae, 18(2), 150-165.

Tuschl, R. J. (1990). From dietary restraint to binge eating: some theoretical considerations. Appetite, 14, 105-109. Vainik, U., Neseliler, S., Konstabel, K., Fellows, L. K., \& Dagher, A. (2015). Eating traits questionnaires as a continuum of a single concept. Uncontrolled eating. Appetite, 90, 229-239.

Van den Tol, A. J. M. (2016). The appeal of sad music: A brief overview of current directions in research on motivations for listening to sad music. The Arts in Psychotherapy, 49, 
$44-49$.

Van den Tol, A. J. M., \& Edwards, J. (2013). Exploring a rationale for choosing to listen to sad music when feeling sad. Psychology of Music, 41(4), 440-465.

Van den Tol, A. J. M., \& Edwards, J. (2015). Listening to sad music in adverse situations: How music selection strategies relate to self-regulatory goals, listening effects, and mood enhancement. Psychology of Music, 43(1), 473-494.

Van den Tol, A. J. M., Edwards, J., \& Heflick, N. A. (2016). Sad music as a means for acceptance-based coping. Musicae Scientiae, 20, 1, $68-83$.

Van den Tol, A. J. M., \& Giner-Sorolla, R. (2016). Listening to ironically-enjoyed music: A self-regulatory perspective. Psychology of Music, 30(5), 73 - 89.

Van Goethem, A., \& Sloboda, J. (2011). The functions of music for affect regulation. Musicae Scientiae, 15(2), $208-228$.

Van Strien, T. (2002). The Dutch Eating Behaviour Questionnaire (DEBQ) manual.

Van Strien, T., Cebolla, A., Etchemendy, E., Gutiérrez-Maldonado, J., Ferrer-García, M., Botella, C., \& Baños, R. (2013). Emotional eating and food intake after sadness and joy. Appetite, 66, 20-25.

Van Strien, T., Frijters, J. E. R., Bergers, G. P. A., \& Defares, P. B. (1986). The Dutch eating behavior questionnaire (DEBQ) for assessment of restrained, emotional, and external eating behavior. International Journal of Eating Disorders, 5(2), 295-315.

Wardle, W. (1987). Eating style: A validation study of the Dutch eating behaviour questionnaire in normal subjects and women with eating disorders. Journal of Psychosomatic Research, 31(2), 161-169.

Wilfley, D. E., Schwartz, M. B., Spurrell, E. B., \& Fairburn, C. G. (1997). Assessing the specific psychopathology of binge eating disorder patients: interview or self-report?Behaviour Research and Therapy, 35(12), 11511159.

World Health Organization. (2016). The ICD-10 classification of mental and behavioural disorders: Clinical descriptions and diagnostic guidelines. Geneva, Switzerland: World Health Organization. 


\section{Endnotes}

1) Depression levels which were 1 SD below the mean were outside the range of the scale and therefore replaced by 1 . 
Table 1

Descriptive Statistics for Age, BMI, and each Sub-scale, as a Total, According to Gender, and Compared Across Gender.

\begin{tabular}{|c|c|c|c|c|c|c|}
\hline & & Mean (SD) & & $\begin{array}{c}\text { Difference } \\
\text { Scores } \\
\text { Across } \\
\text { Gender }\end{array}$ & $p$-value & Partial $\eta^{2}$ \\
\hline & $\begin{array}{l}\text { Total } \\
(N=571)\end{array}$ & $\begin{array}{c}\text { Male } \\
(N=188)\end{array}$ & Female $(N=376)$ & & & \\
\hline \multicolumn{7}{|l|}{ Person } \\
\hline Age & $23.18(4.87)$ & $23.41(4.38)$ & $23.05(5.11)$ & .36 & .41 & .00 \\
\hline Body Mass Index & $24.04(6.13)$ & $24.55(4.58)$ & $23.75(6.79)$ & .80 & .10 & .00 \\
\hline \multicolumn{7}{|c|}{ Music in Mood Regulation Scale } \\
\hline Entertainment & $3.32(.83)$ & $4.09(.83)$ & $4.20(.73)$ & .13 & .10 & .00 \\
\hline Strong- Sensation & $3.63(.79)$ & $3.64(.77)$ & $3.63(.80)$ & .00 & .07 & .00 \\
\hline Revival & $3.32(.85)$ & $3.35(.82)$ & $3.30(.82)$ & .05 & .07 & .00 \\
\hline Diversion & $3.25(.86)$ & $3.17(.87)$ & $3.30(.85)$ & .13 & .08 & .00 \\
\hline Discharge & $2.81(.94)$ & $2.68(.92)$ & $2.87(.94)$ & .18 & .08 & .01 \\
\hline Mental Work & $3.38(.89)$ & $3.32(.90)$ & $3.41(.87)$ & .10 & .08 & .00 \\
\hline Solace & $3.16(.89)$ & $3.06(.88)$ & $3.21(.89)$ & .15 & .08 & .01 \\
\hline \multicolumn{7}{|c|}{ The Dutch Eating Behaviour Questionnaire } \\
\hline Emotional Eating & $2.50(.80)$ & $2.23(.71)^{*}$ & $2.63(.81)$ & .40 & .000 & .05 \\
\hline \multicolumn{7}{|c|}{ Depression, Anxiety, and Stress Scales } \\
\hline Depression & $1.68(.72)$ & $1.53(.57)$ & $1.75(.77)$ & .22 & .06 & .02 \\
\hline Anxiety & $1.66(.61)$ & $1.54(.56)$ & $1.66(.63)$ & .12 & .05 & .01 \\
\hline Stress & $1.92(.72)$ & $1.73(.60)^{*}$ & $2.02(.75)$ & .29 & .000 & .04 \\
\hline
\end{tabular}

Note. SD refers to the Standard Deviation of the mean. $N$ refers to the number of participants. $p$-value refers to the 2 tailed significance levels of the t-test conducted to compare means across genders. Partial $\eta^{2}$ refers to the effect size of the $95 \%$ confidence interval for each identified difference, giving 0.01 (small), 0.09 (medium) and 0.25 (large) values. * denotes a significant gender difference at $p<.0045$. EE items are rated from 1 (Never) to 5 (Very Often) scale. Music in Mood Regulation items (see text for sub-scales) are rated from 1 (Strongly Disagree) to 5 (Strongly Agree). DASS items (see text for sub-scales) are rated from 1 (did not apply at all) to 4 (applied very much, or most of the time). 
Table 2a

Relationships between EE, Stress, Anxiety and Depression and all Music Listening Strategies measured as Regression Coefficients Controlled for Gender and BMI where relevant.

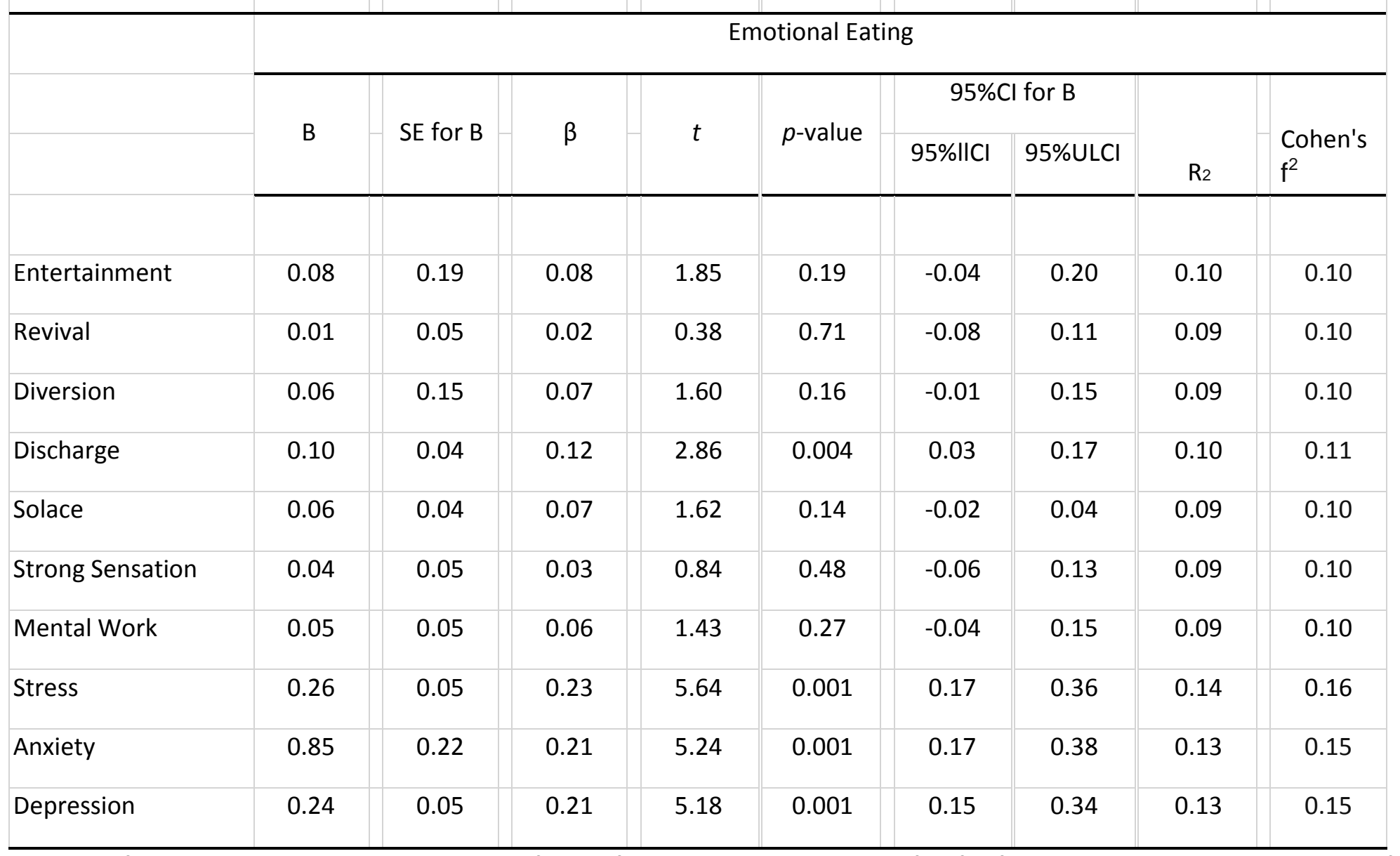

Note: B refers to unstandardized Beta value. SE for B refers to the standard error of B. $B$ refers to standardized Beta value. $t$ refers to $B$ divided by its own standard error. $p$ value refers to the 2 tailed significance levels of the regression-coefficient. $95 \% \mathrm{Cl}$ for $\mathrm{B}$ refers to Confidence Interval for B measured at 95 percent and based on a1000 bias corrected bootstraps, with $95 \% \mathrm{LLCl}$ referring to the Lower Limit, and $95 \% \mathrm{ULCl}$ referring to the Upper Limit. Cohen's $\mathrm{f}^{2}$ refers to the effect size of the regression analysis and is interpreted as $0.02=$ small, $0.15=$ medium, $0.35=$ large. Regression coefficients are controlled for gender and BMI. Further analyses of the data did however reveal that when analyses controlled for gender and BMI were significant, these were also significant when not controlled for either (or both) of these variables. 
Table 2b: Relationships between EE, Stress, Anxiety and Depression and all Music Listening Strategies measured as Regression Coefficients Controlled for Gender and BMI where relevant.

\begin{tabular}{|c|c|c|c|c|c|c|c|c|c|}
\hline & \multirow[b]{2}{*}{ B } & \multirow[b]{2}{*}{ SE for B } & \multirow[b]{2}{*}{$\beta$} & \multirow[b]{2}{*}{$t$} & \multirow[b]{2}{*}{$p$-value } & \multicolumn{2}{|c|}{$95 \% \mathrm{Cl}$ for $\mathrm{B}$} & \multirow[b]{2}{*}{ R2 } & \multirow[b]{2}{*}{ Cohen's f } \\
\hline & & & & & & $95 \% \| \mathrm{ICl}$ & $95 \%$ ULCI & & \\
\hline \multicolumn{10}{|l|}{ DV:Stress } \\
\hline Entertainment & 0.03 & -0.04 & 0.03 & 0.77 & 0.49 & -0.06 & 0.11 & 0.05 & 0.05 \\
\hline Revival & -0.00 & 0.04 & -0.00 & -0.07 & 0.94 & -0.08 & 0.07 & 0.05 & 0.05 \\
\hline Diversion & 0.07 & 0.04 & 0.09 & 2.03 & 0.07 & -0.008 & 0.15 & 0.06 & 0.06 \\
\hline Discharge & 0.22 & 0.03 & 0.28 & 6.99 & 0.001 & 0.15 & 0.28 & 0.13 & 0.14 \\
\hline Solace & 0.12 & 0.03 & 0.15 & 3.64 & 0.001 & 0.05 & 0.19 & 0.07 & 0.08 \\
\hline Strong Sensation & 0.15 & 0.04 & 0.16 & 3.93 & 0.001 & 0.07 & 0.23 & 0.07 & 0.08 \\
\hline Mental Work & 0.05 & 0.04 & 0.2 & 4.87 & 0.26 & -0.26 & 0.14 & 0.09 & 0.10 \\
\hline \multicolumn{10}{|l|}{ DV: Anxiety } \\
\hline Entertainment & 0.02 & 0.03 & 0.06 & 1.52 & 0.66 & -0.06 & 0.09 & 0.00 & 0.00 \\
\hline Revival & 0.02 & 0.03 & 0.05 & 1.12 & 0.49 & -0.04 & 0.09 & 0.00 & 0.00 \\
\hline Diversion & 0.05 & 0.03 & 0.08 & 1.83 & 0.10 & -0.01 & 0.12 & 0.01 & 0.06 \\
\hline Discharge & 0.17 & 0.03 & 0.27 & 6.55 & 0.001 & 0.12 & 0.23 & 0.07 & 0.07 \\
\hline Solace & 0.09 & 0.03 & 0.14 & 3.26 & 0.001 & 0.03 & 0.16 & 0.02 & 0.02 \\
\hline Strong Sensation & 0.06 & 0.03 & 0.16 & 3.96 & 0.08 & -0.01 & 0.12 & 0.03 & 0.01 \\
\hline Mental Work & 0.12 & 0.03 & 0.21 & 5.23 & 0.001 & 0.06 & 0.17 & 0.05 & 0.01 \\
\hline
\end{tabular}




\begin{tabular}{|c|c|c|c|c|c|c|c|c|c|}
\hline DV_:Depression & & & & & & & & & \\
\hline Entertainment & 0.06 & 0.05 & 0.04 & 0.095 & 0.20 & -0.04 & 0.15 & 0.03 & 0.00 \\
\hline Revival & 0.04 & 0.04 & 0.04 & 1.02 & 0.31 & -0.04 & 0.12 & 0.03 & 0.00 \\
\hline Diversion & 0.07 & 0.04 & 0.09 & 2.04 & 0.06 & -0.00 & 0.32 & 0.04 & 0.01 \\
\hline Discharge & 0.26 & 0.03 & 0.32 & 8.07 & 0.001 & 0.19 & 0.32 & 0.13 & 0.11 \\
\hline Solace & 0.16 & 0.04 & 0.19 & 4.57 & 0.001 & 0.08 & 0.23 & 0.06 & 0.04 \\
\hline Strong Sensation & 0.15 & 0.04 & 0.04 & 4.07 & 0.001 & 0.06 & 0.23 & 0.06 & 0.03 \\
\hline Mental Work & 0.17 & 0.04 & 0.21 & 4.97 & 0.001 & 0.11 & 0.25 & 0.06 & 0.05 \\
\hline
\end{tabular}

Note: B refers to unstandardized Beta value. SE for B refers to the standard error of B. $B$ refers to standardized Beta value. $t$ refers to $B$ divided by its own standard error. $p$ value refers to the 2 tailed significance levels of the regression-coefficient. $95 \% \mathrm{Cl}$ for B refers to Confidence Interval for B measured at 95 percent and based on a1000 bias corrected bootstraps, with $95 \% \mathrm{LLCl}$ referring to the Lower Limit, and $95 \% \mathrm{ULCl}$ referring to the Upper Limit. Cohen's $\mathrm{f}^{2}$ refers to the effect size of the regression analysis and is interpreted as $0.02=$ small, $0.15=$ medium, $0.35=$ large. Regression coefficients are controlled for gender and BMI. Further analyses of the data did however reveal that when analyses controlled for gender and BMI were significant, these were also significant when not controlled for either (or both) of these variables. 
Table 3

An Overview of the effects of Music Listening Strategies on EE for Different Levels of Depression, Anxiety, or Stress.

\begin{tabular}{|c|c|c|c|c|c|c|c|c|c|}
\hline & & & DV: E & onal Ea & & & & & \\
\hline & $\mathrm{B}$ & $\begin{array}{c}\text { SE for } \\
\text { B }\end{array}$ & $\mathrm{t}$ & $\begin{array}{c}p \text { - } \\
\text { value }\end{array}$ & $\begin{array}{c}95 \% 11 C I \\
\text { for B }\end{array}$ & $\begin{array}{l}95 \% \text { ULCI } \\
\text { for B }\end{array}$ & $\begin{array}{l}\mathrm{R}^{2} \\
\text { for } \\
\text { step } \\
2\end{array}$ & $\begin{array}{l}\text { adjusted } \\
\mathrm{R}^{2} \text { for } \\
\text { step } 2\end{array}$ & $\begin{array}{c}\mathrm{R}^{2} \\
\text { change } \\
\text { between } \\
\text { step } 1 \& 2\end{array}$ \\
\hline Entertainment- Depression & -0.13 & 0.05 & -2.53 & 0.012 & -0.23 & -0.03 & 0.15 & 0.14 & 0.01 \\
\hline Low Depression & 0.17 & 0.05 & 2.99 & 0.003 & 0.06 & 0.27 & & & \\
\hline High Depression & -0.01 & 0.05 & -0.25 & 0.803 & -0.12 & 0.09 & & & \\
\hline Diversion- Depression & -0.16 & 0.05 & -3.35 & 0.001 & -0.25 & -0.06 & 0.15 & 0.14 & 0.02 \\
\hline Low Depression & 0.16 & 0.05 & 3.20 & 0.001 & 0.06 & 0.26 & & & \\
\hline High Depression & -0.06 & -0.06 & -1.17 & 0.242 & -0.15 & 0.04 & & & \\
\hline Mental work- Depression & -0.13 & 0.05 & -2.69 & 0.007 & -0.23 & -0.04 & 0.14 & 0.14 & 0.01 \\
\hline Low Depression & 0.10 & 0.05 & 2.03 & 0.042 & 0.00 & 0.19 & & & \\
\hline High Depression & -0.08 & 0.05 & -1.63 & 0.103 & -0.2 & 0.02 & & & \\
\hline Entertainment- Stress & -0.13 & 0.05 & -2.47 & 0.013 & -0.23 & -0.03 & 0.15 & 0.15 & 0.01 \\
\hline Low Stress & 0.16 & 0.06 & 2.98 & 0.003 & 0.06 & 0.27 & & & \\
\hline High Stress & -0.02 & 0.06 & -0.38 & 0.706 & -0.13 & 0.09 & & & \\
\hline Diversion- Stress & -0.14 & 0.05 & -2.85 & 0.004 & -0.23 & -0.04 & 0.15 & 0.15 & 0.01 \\
\hline Low Stress & 0.15 & 0.05 & 2.89 & 0.004 & 0.05 & 0.25 & & & \\
\hline High Stress & -0.05 & 0.05 & -0.95 & 0.341 & -0.15 & 0.05 & & & \\
\hline Mental work- Stress & -0.1 & 0.05 & -2.14 & 0.033 & -0.20 & -0.01 & 0.15 & 0.14 & 0.01 \\
\hline Low Stress & 0.08 & 0.05 & 1.64 & 0.101 & -0.02 & 0.18 & & & \\
\hline High Stress & -0.07 & 0.05 & -1.27 & 0.203 & 0.04 & -0.01 & & & \\
\hline
\end{tabular}

Note: B refers to the Unstandardized Beta coefficient. SE refers to the standard error of B. $t$ refers to Unstandardized Beta coefficient divided by its own standard error. $p$-value refers to the 2 tailed significance levels of the of the moderation analysis. 95\%LLCC refers to Lower Level Confidence Interval of the Beta value at 95 percent. 95\% ULCC refers to Lower Level Confidence Intervals of the Beta value at 95 percent. $\mathrm{R}^{2}$ for step 2 and adjusted R ${ }^{2}$ for step 2 refers to the effect size of the overall model at step $2 . \mathrm{R}^{2}$ change between step $1 \& 2$ refers to the increase in effect size for the overall model when the interaction is included. 
Figure 1: Mean scores on EE for Depression and Entertainment, Controlled for Gender and BMI.

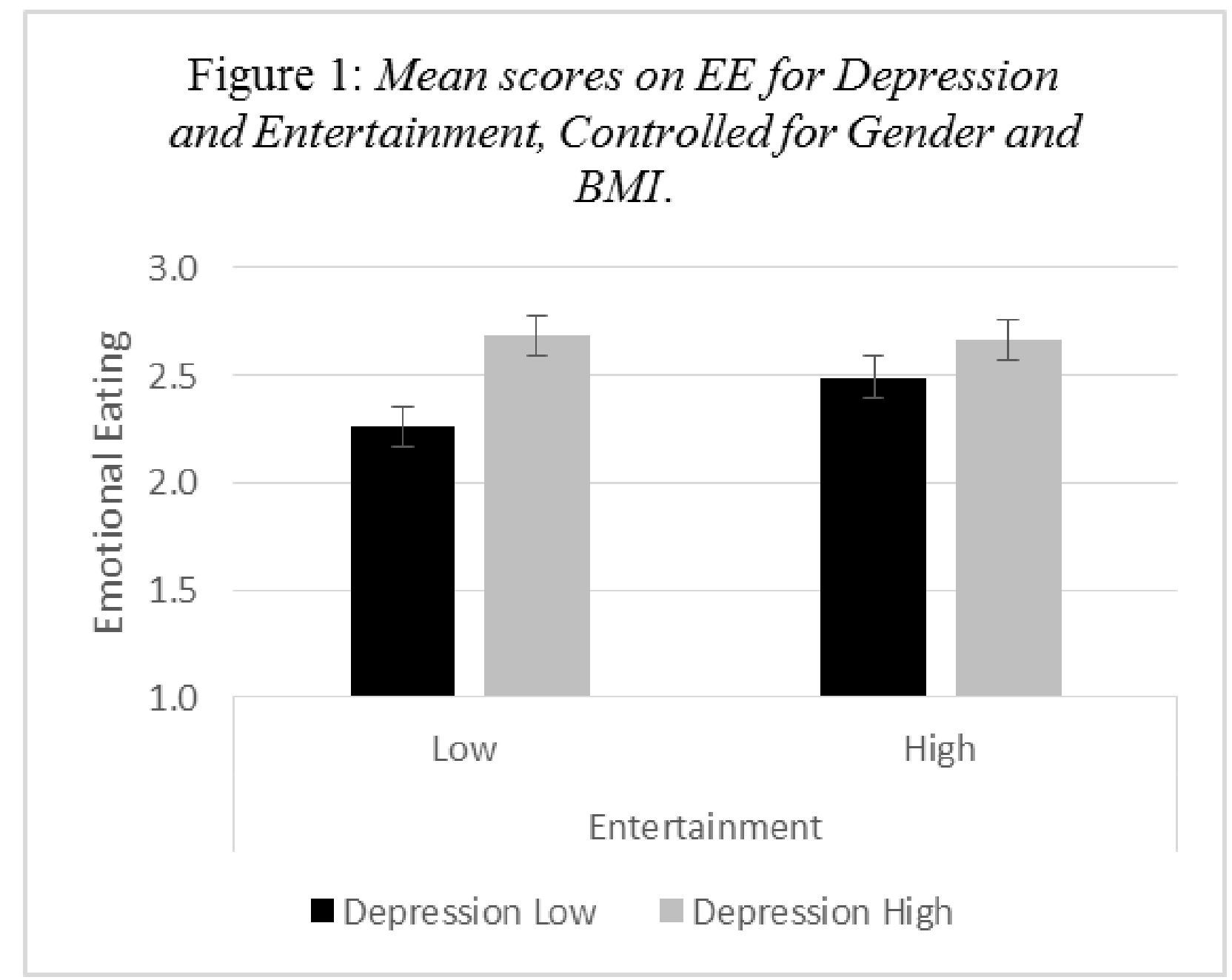

